Herzschr Elektrophys 2015 $\cdot 26: 307-308$

DOI 10.1007/s00399-015-0408-0

Online publiziert: 26. November 2015

(c) Springer-Verlag Berlin Heidelberg 2015

CrossMark

\section{Carsten W. Israel ${ }^{1} \cdot$ Lars Eckardt $^{2}$}

${ }^{1}$ Klinik für Innere Medizin - Kardiologie, Diabetologie \& Nephrologie,

Evangelisches Krankenhaus Bielefeld, Bielefeld, Deutschland

${ }^{2}$ Abteilung für Rhythmologie, Department für Kardiologie und Angiologie,

Universitätsklinikum Münster, Münster, Deutschland

\title{
Komplikationsmanagement in der invasiven Elektrophysiologie
}

In dieser Ausgabe von Herzschrittmachertherapie + Elektrophysiologie sind wieder exzellente Originalbeiträge, Kasuistiken, eine Elektrophysiologie-Weiterbildung und das deutsche Herzschrittmacherund ICD-Register enthalten, für die ich mich bei den Autoren sehr herzlich bedanken möchte. Ein besonderer Dank gilt dabei Herrn Markewitz, der mit der Zuverlässigkeit und Pünktlichkeit eines Schweizer Uhrwerks die Registerdaten (rund 150.000 Schrittmacher- und ICDImplantationen in Deutschland) Jahr für Jahr analysiert, besondere Aspekte beleuchtet und in einen europäischen Kontext stellt.

Als Schwerpunkt wird das Thema „Probleme, Komplikationen und Notfälle der invasiven Elektrophysiologie“ betrachtet, das wir zuletzt im Dezember 2007 aufgegriffen hatten. Warum wurde dieses Thema jetzt wieder aufgenommen?

Nach 8 Jahren ist ein Update notwendig. Wir haben gelernt, dass sogar Leitlinien keine in Stein gemeißelten Gesetze darstellen, sondern alle paar Jahre erneuert werden müssen. In der Elektrophysiologie hat sich in den letzten 8 Jahren (erfreulich) viel getan: Ablationsverfahren, die vor 10 Jahren noch das Etikett „experimentell“ hatten oder die es noch gar nicht gab, stellen heute Routineverfahren dar mit einem „Klasse-IA-Label“ in den Leitlinien. Dies wird in den Beiträgen von Steven et al. und Wasmer et al. deutlich, in denen auf linkskardiale Ablationen wie die Pulmonalvenenisolation und Ablation linksventrikulärer Arrhythmien eingegangen wird, beides heute - trotz des Komplikationspotenzials - etablierte Verfahren.
Bei der Device-Implantation hat sich insbesondere die CRT-Implantation von einem Exoten zu einem RoutineVerfahren mit einer deutlichen zahlenmäßigen Zunahme entwickelt. Im Jahr 2007 wurden in Deutschland 99 CRTSysteme pro 1 Mio. Einwohner implantiert. Diese Zahl lag 2014 bei 242 (davon $83 \%$ CRT-D-Systeme), was einen Anstieg um 144\% bedeutet. Ebenso wurde es - leider - in den letzten 10 Jahren erforderlich, sich besondere Gedanken zur Elektrodenextraktion zu machen, da dieser Eingriff immer häufiger notwendig wurde. Mit Herrn Burger konnten wir zu diesem Thema einen der deutschlandweit erfahrensten Extrakteure gewinnen, der aus seiner reichen Erfahrung zahlreiche Fälle und Tipps für das Verhalten im Notfall (und die Vermeidung dieser Situation) geben kann.

Bei der primärprophylaktischen Implantation von Defibrillatoren haben viele Zentren bereits vor Publikation der SIMPLE- und NORDIC-Studie begonnen, auf eine Defibrillationstestung $\mathrm{zu}$ verzichten. Inwieweit dies gerechtfertigt oder sogar sinnvoll ist, wird in der Übersicht des kanadischen Teams unter Jeff Healey, das die SIMPLE-Studie leitete, dargestellt. Wie im zweiten Teil der Analyse des deutschen Device-Registers in dieser Ausgabe von Herzschrittmachertherapie + Elektrophysiologie ausgeführt, wurden in Deutschland rund 50.000 ICDs implantiert, davon über $70 \%$ primärprophylaktisch. Die Frage, ob eine Defibrillationstestung durchgeführt werden soll oder nicht, stellt sich in Deutschland also jedes Jahr über $30.000 \mathrm{Mal}$.
Die häufigste Device-Implantation stellt weiterhin die Schrittmacherimplantation dar. Wie Markewitz im ersten Teil der Analyse des deutschen Herzschrittmacher-Registers in dieser Ausgabe ausführt, wurden 2013 in rund 1000 deutschen Zentren mehr als 100.000 Schrittmacher implantiert, ausgetauscht oder revidiert. Dass auch bei dieser Prozedur, die ja seit Jahrzehnten zum basalen Standard-Repertoire der invasiven Kardiologie gehört, unerwartete signifikante Probleme und Komplikationen auftreten können, wird in einem hier$\mathrm{zu}$ verfassten Beitrag dargestellt. Eine Schrittmacherimplantation, die sich als schwierig und problematisch erweist, beginnt meist mit einem erschwerten $\mathrm{Zu}$ gang, z. B. wenn nach endloser erfolgloser Subklaviapunktion die Vena cephalica präpariert wird und sich dann erst ein Subklaviaverschluss, eine Venenanomalie oder eine atypische Lage zeigt. Wie man bereits beim venösen Zugang den Verlauf der gesamten Implantation angenehmer gestalten kann, wird in den Fokus der Ausführungen gestellt. Dabei werden auch seltene Probleme und Notfälle des Zugangs und deren schnelle Lösung (und zukünftige Vermeidung) dargestellt. Die Erläuterung dieser Probleme soll helfen, dass bei deren Auftreten der Puls des Implanteurs nicht tachykard wird und kein plötzlicher Adrenalinstoß das logische Denken behindert.

Die invasive Elektrophysiologie-Landschaft 2015 sieht anders aus als 2007: andere Prozeduren, andere Materialien, andere Techniken. Vor allem aber finden sich an sehr vielen Orten Menschen, die zwar seit einigen Jahren invasive elektro- 
physiologische Prozeduren durchführen, dies aber 2007 noch nicht getan haben. Für alle diese Kolleginnen und Kollegen soll dieses Heft eine Hilfe darstellen, die Angst vor Komplikationen und Notfällen $\mathrm{zu}$ reduzieren und einen Plan zu entwickeln, was zu tun ist, bevor das Problem eines Tages tatsächlich auftritt.

Die Statistik hat immer Recht, und daher werden auch seltene Komplikationen und Notfälle irgendwann auftreten, wenn jemand viele Prozeduren durchführt. Das Schwerpunktthema über Probleme, Komplikationen und Notfälle soll dabei helfen, eine Kultur der Diskussion von Fehlern zu fördern, denn aus Fehlern lernt man bekanntlich besonders gut. Es ist sinnvoll, wenn ein Fehler nur einmal gemacht wird und möglichst viele daraus lernen und ihn dadurch vermeiden können. Aber nur wenn Ärzte bereit sind, über eigene Fehler zu berichten, kann eine solche Kultur mit allen Vorteilen für Ärzte und Patienten wachsen. Oft haben nur Kollegen, die aufgrund ihrer Expertise über jeden Zweifel erhaben sind, ein so breites Kreuz, dass sie über eigene Fehler berichten können. Es wäre wünschenswert, dass mehr Personen, die eher dem Normalfall der invasiv tätigen Ärzte entsprechen, über typische Probleme, Komplikationen und Notfälle ihres Alltags berichten, ohne Angst vor negativen Konsequenzen. Die Arbeit mit Registern ist hierbei ein Anfang. Jedoch ist diese zentral davon abhängig, ob die Fälle, die "nicht geklappt“ haben, auch eingeschlossen und nicht unter den Teppich gekehrt wurden. Ansonsten können Register eine irreführend niedrige oder komplett fehlende Rate an Komplikationen berichten, die dazu führt, dass jeder seine eigenen Komplikationen auch lieber verheimlicht, um nicht schlecht dazustehen. Diese falsche Kultur schadet der Weiterentwicklung, der Fortbildung, dem Patienten und auch dem Arzt, der aus Angst vor Fehlern im Sinne einer "self-fulfilling prophecy“ genau diese eher macht.

In diesem Sinne wünschen wir allen Lesern viel Spaß bei der Lektüre dieser Ausgabe von Herzschrittmachertherapie + Elektrophysiologie. Mögen die vielen Tipps und Tricks im Alltag helfen und diesen auch im Winter freundlich strahlend statt grau und trübe machen.

Ihre,

Carsten W. Israel

Lars Eckardt

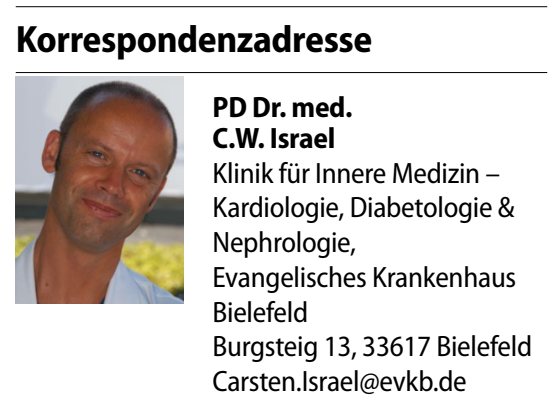

Interessenkonflikt. C.W. Israel und L. Eckardt geben an, dass kein Interessenkonflikt besteht. 